\title{
An Architecture for Recommendation of Courses in E-learning System
}

\author{
Bhupesh Rawat \\ Computer Science Department of Babasaheb Bhimrao Ambedkar University, Lucknow, 226025, India \\ E-mail: bhr222@gmail.com \\ Sanjay K. Dwivedi \\ Computer Science Department of Babasaheb Bhimrao Ambedkar University, Lucknow, 226025, India \\ E-mail: skd200@yahoo.com
}

\begin{abstract}
Over the last few years, the face of traditional learning has changed significantly, due to the emergence of the web. Consequently several learning systems have emerged such as computer-based learning, web-based learning among others, meeting different kinds of educational needs of the learners and educators as well. E-learning systems allow educators, distribute information, create content material, prepare assignments, engage in discussions, and manage distance classes among others. They accumulate a huge amount of data as a result of learner's interaction with the site. This data can be used to find students' learning pattern based on which appropriate courses could be recommended to them. However existing approaches of recommending courses to learner offer the same course to all the learners irrespective of their knowledge and skill level which results in decreasing their academic performance. This paper proposes an architecture for the recommendation of courses to a learner based on his/her profile. The profile of a learner is created by applying k-means algorithm to learner's interaction data in moodle. The results show that the non active learners should not be recommended advanced courses if they have obtained poor marks and are not active in the concern course. In the initial stage we discover learners' performance in data mining course which will further be extended to other courses as well.
\end{abstract}

Index Terms-Data mining, Weka, Moodle, Data preprocessing, E-learning, Educational data mining.

\section{INTRODUCTION}

Educational data mining (EDM), involves developing methods that discover knowledge from data originating from educational environments[1].The research community has shown growing interest in the application of data mining techniques in education[2][4] [22][23][24][25][26][27][28][29]. Data Mining (DM), also known as knowledge discovery in database (KDD) refers to the process of nontrivial extraction of implicit, previously unknown, and potentially useful information from huge amount of data in the database [6]. It has been used successfully in many areas including education, banking, telecommunication, business among others.
Data mining includes tasks such as outlier detection, association rule mining, clustering, classification, regression etc [2]. It provides several techniques such as Apriori, K-means, Sequential mining and many more which can be used to discover knowledge about learner's behavior in order to improve the overall learning process in the e-learning system.

Recommending learning resources to learners based on their personal characteristics has been an area of research by many researchers. In one such work [36] the authors have uses Bayesian networks of courses relationships. The method uses feedback of learning resources from the learners and examination results of training course as data sample. The method is integrated with RFM model in order to classify the students and help them make better decisions. Experimental results show the effectiveness of the approach.

Predicting student's performance timely can help them to improve their learning process, consequently improving student academic performance. Keeping this in mind the authors in [35] employed some of the data mining techniques such as Naïve Bayes, Neural network and decision tree and found that Naïve Bayes classifiers outperform the other two classifier by achieving overall prediction accuracy of $86 \%$.

This paper proposes an architecture for the recommendation of courses to learners based on their profile which is built by using k-means algorithm. The proposed architecture is to be implemented in moodle. Moodle (modular object oriented developmental learning environment)is one of the most widely used open source learning management systems. They can record any student activities including reading, writing, taking tests, performing various tasks, and even communicating with peers through collaborative forums such as chat, forum, glossary, wiki and workshop. They normally provide a database that stores all the system's information such as personal information about the users (profile), academic results and users' interaction data (usage data). However, due to large amount of data generated by these systems, it is very difficult to manage it manually by the administrator and instructor as well [1]. Recommender systems use several techniques including data mining, agent and reasoning among others to recommend items 
based on the profile of a user.

However most of the existing e-learning systems offer the same courses to all the learners regardless of their level of knowledge, skill and interest. Hence it becomes necessary to recommend learning material that best matches users learning goal. For example a learner who has not done well in quizzes in a particular courses and has not completed any assignment and his/her level of activity in forums, chat and other collaborative platforms within moodle has been very low, should be categorized as non active students and should be recommended learning material in such a way that help him to improve his academic performance. Moreover recommending advanced topics of a course to a learner in which he has not performed well would not serve the purpose of enhancing his learning process which in turn leads to further deteriorating his academic performance.

The remaining part of the paper is organized as follows: First we review related work in recommendation of courses in e-learning system. Then we describe the architecture of the learning resource recommendation system. Next, we discuss data preprocessing which is an essential step and helps us in making data suitable for data mining algorithms. Then we present and discuss the results. Finally, we discuss the conclusion and future work section.

\section{RELATED WORK}

A significant amount of work is currently underway on recommendation system which recommends learning material to a learner. Some of the key works is presented here. In [8] they have presented an e-learning ITS to recommend feasible learning courseware for individual student. They have also discussed the structure of curriculum and how to design object oriented learning courseware based on sharable content object reference model. A fuzzy approach is also presented in order to explain cognitive ability level. In [12] they have proposed a recommender system consisting of two modules. The first module classifies the students as good and average. After this classification the second module recommends courses based on the resources which are accessed by majority of good students. In another work [13] they discussed the current state of research and application of data mining methods in e-learning. They also discussed various e-learning problems to which the data mining techniques have been applied such as learner's classification based on their learning performance, discovery of irregular learning behavior, clustering based on similar E-learning system usage and system adaptability according to students' requirements.

In yet another work the authors in [14] introduced a prototype for an adaptive navigation system which uses a dissimilarity measure between student and exercise profiles to rank and recommend exercises. In [15] they narrated technical activity based course recommender system in which they defined an architectural model of this method using a collaborative filtering technique which is based on collecting and analyzing information about user activity.

In [3] the authors have presented a learning resource recommendation framework using rule based reasoning approach which allows instructors and students to treat learning resources as learning objects which also utilizes ontology for searching and reusing learning objects. In [9] they proposed a generic architecture for developing educational recommender system which is independent of the type of recommendation generated. Their recommendation is based on user profile, their preferences and user's objective.

In [4] they described a personalized recommender system that uses web mining technique for recommending a student which link to visit next within an adaptable educational hypermedia system. They used data mining techniques such as clustering and sequential pattern mining together for discovering personalized recommendation links. In [5] they have proposed a cloud based architecture for a system that recommends learning resources based on the affective state of a learner. In [10] they have presented an adaptive e-learning recommender system which recommends resources to learner according to their interest and level of learners. The system's design is based on IEEE LTSA which is learning technology system architecture. They have also proposed a feedback extractor with fusion capability in order to combine multiple feedback measures to infer user preferences.

In [11] they have proposed an adaptive and intelligent web based programming tutoring system-protus which uses recommendation and adaptive hypermedia techniques. This system automatically guides the learner's activities and recommends relevant links and action to learner during the learning process. The authors in [16] introduced an evolving web-based learning systm which can adapt itself not only to the varying requirement of its user but also to the open web. In [18] they presented have used classification algorithm in order to help in improving the quality of higher educational system by evaluating student data to study the main attributes that may affect the student's performance. They have also used CRISP framework for data mining of students' academic data. In [7] they have presented a survey which proposes two techniques called a fuzzy tree structure learning activity model and a learner profile model. These two methods comprehensively explain the difficult learning activities and learner profiles in order to learn the semantic relation between learning activities or learner requirements of each individual learner.

In [19] the authors suggested a framework for a personalized learning recommender system with the goal of helping students find learning materials, they might need to read. In another work, the authors suggested [20] how the changing preferences of learner over time can be tracked with adaptive machine learning algorithms. In this approach, they begin by building an initial decision model based on learning style using the available student's data. This model was further updated with the data generated by student's interaction with the system in order to reflect more accurately their current preferences. 
In [21] they have proposed a new learning material recommender system based on sequential patter mining and multidimensional attribute based collaborative filtering. They combined the results of two approached using cascade, weighted and mix methods. In [30] they have introduced an e-learning schema that adapts to the learner's ICT (information and communication technology) knowledge level. They have also used IEEE LTSA reference model. Learner's profiles were built by the automatic analysis of responses obtained on a electronic questionnaire which were given to learners. The learner were recommended learning material based based on these profiles.

In [31] they discussed a personalize recommender system which uses web mining techniques for recommending a student which links to visit next within an adaptable educational hypermedia system. In [33] they compare different data mining methods and techniques for classifying students based on their Moodle usage data and the final marks obtained in their respective courses. They also developed a specific mining tool for making the configuration and execution of data mining techniques easier for students.

We propose a recommendation system which recommends courses to learners based on their profile which is created by applying k-means algorithm to learner's transaction data resides in the moodle server.

Our proposed system has the following objectives:

1. To collect data from moodle server on various courses such as data mining, operating system, data structure, database system, computer organization and automata theory. The performance of student in each course is evaluated by taking quizzes, assignments, monitoring learners' activity in collaborative platform such as message chat, forum, and message sent to teacher among others.
2. To perform data preprocessing in order to make data suitable for data mining algorithms.

3. To build learners' profile by applying k-means algorithm.

\section{WEKA (WAIKATO ENVIRONMENT FOR KNOWLEDGE ANALYSIS)}

Weka [32] is data mining software that implements data mining algorithms. It is a collection of machine learning algorithms and data preprocessing tools. It is freely available for download and offers many powerful features (sometimes not found in commercial data mining software). It has become one of the most widely used tools for discovering knowledge from huge amount of data in database. Weka consists of machine learning algorithms for data mining tasks. The algorithms are either applied directly to a dataset or can be called from java source code. Weka implements algorithms for data pre-processing, classification, regression, clustering, and association rules. It also includes a visualization tool which helps in visualizing data in a more comprehensible way. It is written in java and distributed under the terms of GNU general public license. Weka is platform independent. That means it can run almost on any platform whether it is windows or unix. Weka has a range of different algorithms, it provides a common interface for these entire algorithm. There are times when one has to transform a dataset from one form to another required form so weka also includes a variety of tools for performing such tasks. A user can do several tasks such as data preprocessing, execute an algorithms and analyze the results without writing a single line of code in weka. It also includes methods for all the data mining problems such as clustering, association mining, classification and many more.

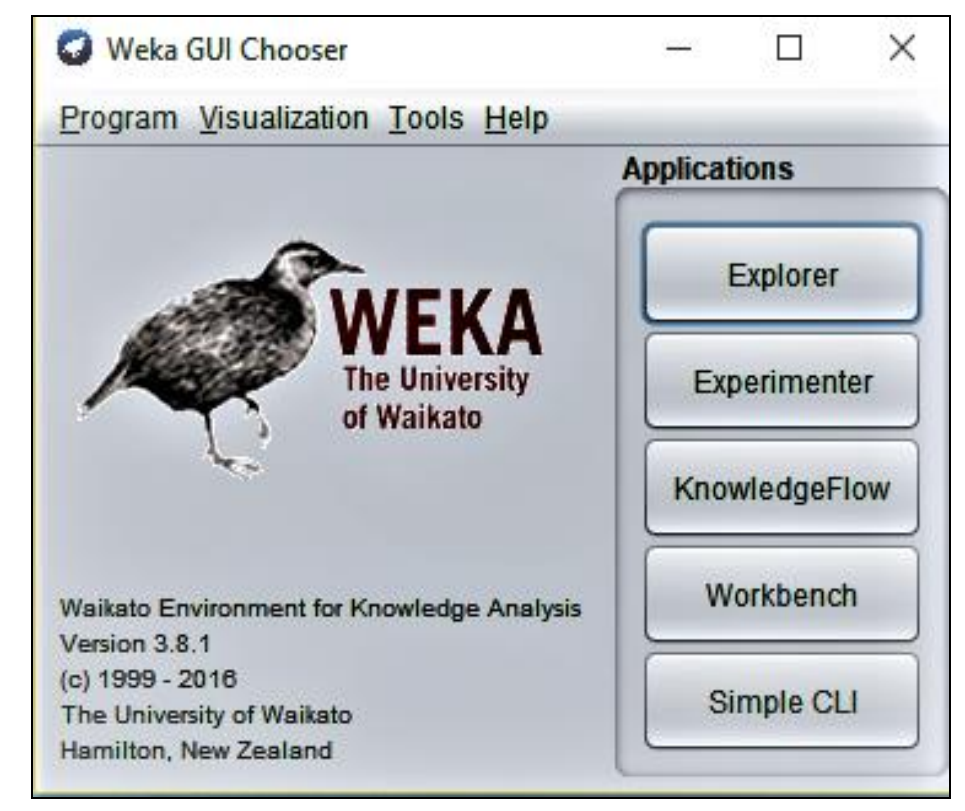

Fig.1. Interface of Weka 3.8.1 


\section{MOOdLE (MODUlar OBJECT ORIENTED DEVELOPMENT LEARNING ENVIRONMENT)}

We use moodle due to its open source nature and being able to run on any platform because of its implementation in java. It allows educators create effective online learning communities [17]. It is used by universities, community colleges, K-12 schools, business and even individual instructors to add web technology to their course. Moodle is freely available on the web at (http://www.moodle.org). Moodle also allows users to have access to its source code and can manipulate it in order to add new functionality to moodle. Moreover, unlike other learning system it records each and every user activity in the log file.
All the components of moodle are modularized such as assignment, quiz, taking a test, adding course etc. Each activity of a learner is recorded in a log file of moodle's server. A $\log$ file collects all the Data including quiz, assignment, chat, forum among others which comes from user's interaction with the site.

The interaction involves any activity done by the user on the site such as click stream data. moodle also allows user to import data in different format such as ARFF,CSV,C4.5 and binary. However weka's native data storage format is ARFF(attribute relation file format) which has two sections including HEADER and DATA. The HEADER section contains details such as the name of relation, name of attribute and its type whereas the data section a set of instances shared by each attribute.

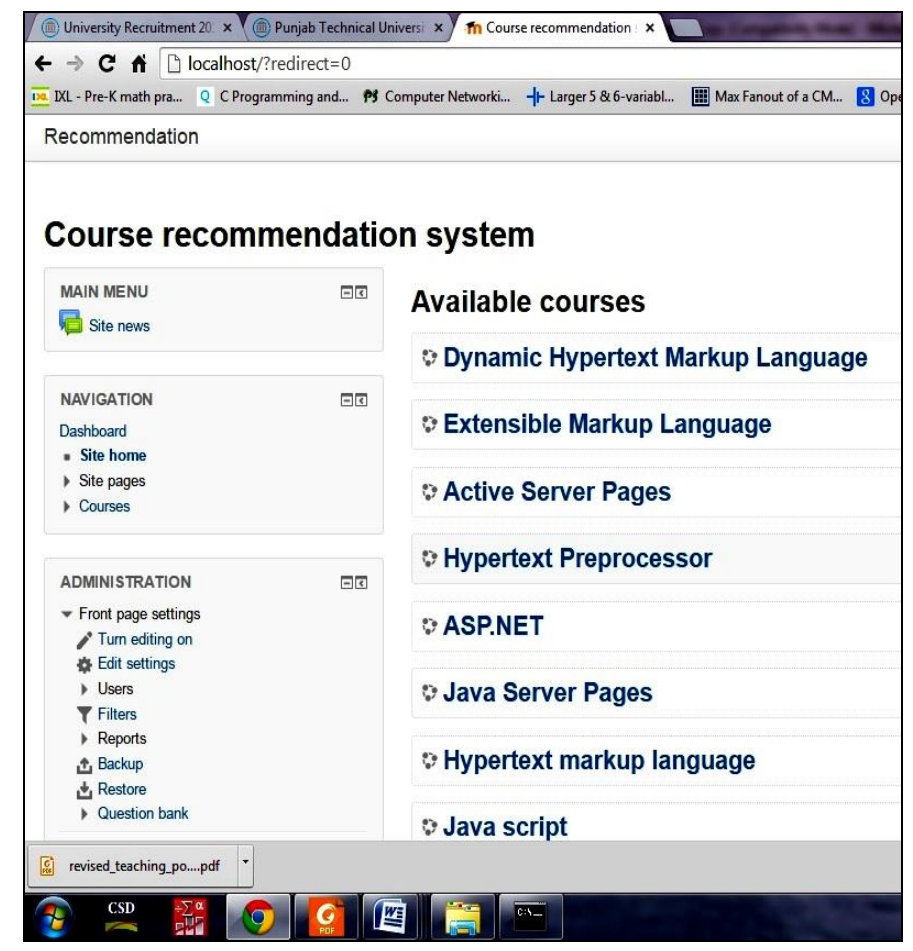

Fig.2. Course Recommendation System

\section{ARChITECTURE OF PROPOSED SYSTEM}

The working of the system shown in Figure. 3 is described below. We provide login to each user in order to ensure that only those users who have authenticated themselves by providing correct user name and password should be able to use the system. If the login information is incorrect then the user is asked again to login. Once the user provided correct login details he/she can use the system. A learner is required to do assignments, quizzes and other activities which involve collaboration activities as well which involves collaboration from other user also such as message sending through chat, message posting and reading in forum, time spent in doing assignment, time spent on forum, number of messages sent to teacher among other activities. All this data gets accumulated in the $\log$ file of moodle's server as a result of user's interaction with the site.

The data is actually stored in relational database such as MYSQL. The technical requirements are quite flexible for installing and running moodle on any platform. The data in database is in raw form and is not suitable for applying data mining algorithms so it needs to be preprocessed. After data preprocessing we are interested in discovering the academic performance of each student in each course by making clusters of students using kmeans algorithm to the preprocessed data.

We use k-means algorithm due to its ability to discover hidden patterns in large amount of data which is required in our case. We are also interested in knowing different groups of students showing similar learning patterns/behavior based on which appropriate learning material could be recommended to them. 


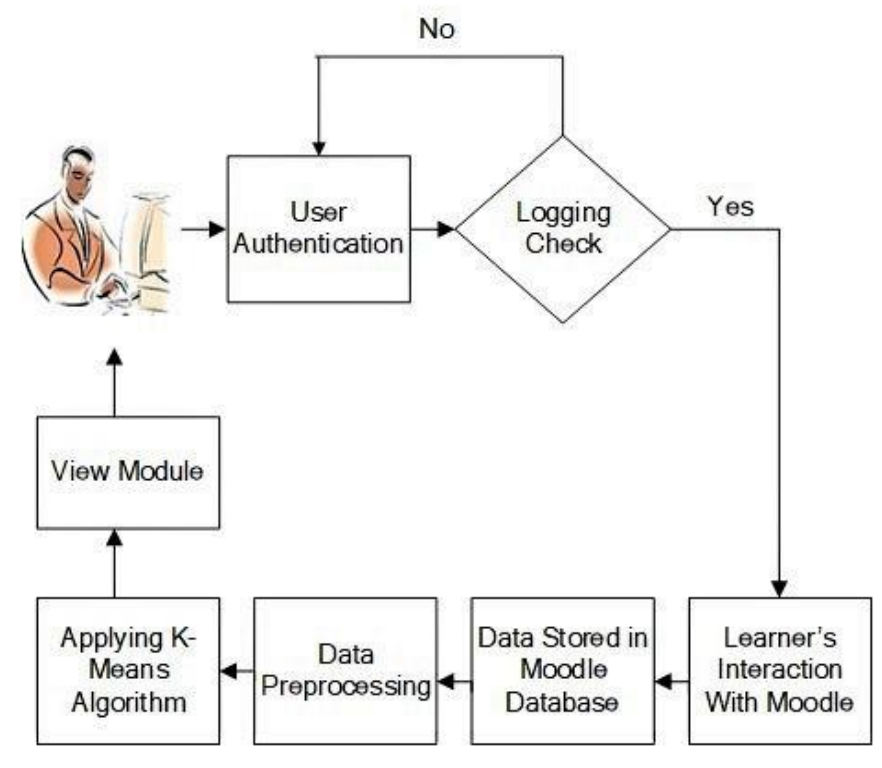

Fig.3. Architecture of Course Recommendation System

The number of clusters produced by k-means depends on the value of $\mathrm{k}$. If we specify $\mathrm{k}=3$ then we get 3 clusters. In our case we have got two clusters. The first cluster is characterized by non active students with very low score in all the activities and quizzes. However the second cluster is characterized by very active students with high score in quizzes and assignments as well.

The architecture is implemented in Moodle. There are many learning management systems which are both commercial and freely available. In Table.1 we can see various attributes used by each student. The attributes considered in our research are C-id, no_assignment_comp,quiz_comp,no_quizpasswd,no_qui z_fail,no_messg_snt_cht,no_msg_teach,msg_snt_forum, Time_assignment,Time_quiz, Time_forum, Mark.

We have considered six courses namely data warehouse and mining, operating system, data structure, software engineering, computer networks and compiler designing which are taught in the department of computer science and engineering of Amrapali group of institute. In order to evaluate the performance of students in each course we have also taken few attributes with their descriptions as shown in Table.1.

Data preprocessing involves several steps including data cleaning, user identification, session identification, path completion, transaction identification, data transformation, data integration and data reduction. However all these steps are not required in our case as moodle provides login to each user which helps us to know the user identification and session identification information.

However the following tasks needs to be performed:

Data selection: There are many courses running in the department of computer science and engineering of the institute of Amrapali, however we have chosen only six courses, including data mining, operating system, data structure, database system, computer organization and automata theory. Corresponding to each course we have used few attributes of each students such as quiz, assignments, message sent by students to teacher, messages sent by students to forum, message sent on chat, time taken by students on quizzes, total time taken by students on forum, total resources access by students, number of resources accessed less than 30 seconds and number of resources visited. We also consider final marks obtained in each course by these students. We have considered a total of 100 students in our research. As the student and course data is spread over several tables in moodle's database so we have considered student and course data as per our requirement.

Create Summarization Table: The summarization table represents information from database at the required level (student). As usage data is spread over several table hence a summarization table has been created in order to represent information for our objective. The summarization table has a summary per row about all the activities done by each student during the course and the final mark obtained by each student in each course. The summarization table is shown in Table.1.

Data Discretization: It is important for instructor that the data is easy to understand. In other words it should be interpretable and comprehensible. Data discretization helps us to transform numerical data into categorical data. Precise number makes it difficult to comprehend the meaning of data so this step becomes essential. There are few methods for performing data descretization such as manual method in which the user has to specify the cut off points in the mark attributes with four intervals FAIL if value is $<5$; PASS if value is $>5$ and $<7$; GOOD if value is $>7$ and $<9$ and EXCELLENT if value is $>9$. The range of marks specified above may be changed as per the requirements. This transformation of numerical data makes it easier for the instructor to understand the numerical data. We have taken four categories including EXCELLENT, GOOD, AVERAGE and POOR to categorize mark attribute and divide numerical data of all the attribute except course_id to these four categories. For 
example a learner who has attempted all the quizzes (4) where each quiz is on a particular topic of a course and carries 10 marks each and has scored more than 35 out of 40 and has done all the assignments (10) and is very active(the criteria for a student to be active in message chatting is(if no_message_chat>70), spent considerable amount of time in quizzes and has been active on forum, accessed high number of resources and course_total is more than 80 qualifies to be lied in the category of EXCELLENT. Similarly we have defined the criteria for other categories.Another method is the equal-width method which divides the range of the attribute into a fixed number of intervals of equal length. For example the attribute no_message_sent_teacher can be divided into three intervals namely LOW, MEDIUM and HIGH and as per our criteria if number of messages sent by a learner is greater than or equal to 70 then the number of messages sent by a learner to the teacher is HIGH. If it is between 50 and 70 then MEDIUM else it is low.

Transform the Data: After discretization the data must be exported into the required format which is ARFF (attribute relation file format). This format is supported by weka and is suitable for data mining algorithms. ARFF is a text file containing a set of attributes shared by a set of instances. In our case our data was initially in excel file which needs to be converted into CSV file format. Then we transform to ARFF file format.Although data pre-processing includes several sub-steps such as data cleaning, pattern identification, user identification, session identification and path completion, all these steps are not required in our research because user identification and session identification are decided by providing logging to the user. A user provides authentication details before logging into the system and he/she also logs out once he finished

Table.1. Attribute Shared by Each Learner

\begin{tabular}{|c|c|}
\hline Attribute name & Description \\
\hline c_id & Course identification \\
\hline assign_comp & Assignments completed \\
\hline no_quiz_comp & Number of quizzes completed \\
\hline no_quiz_passd & Number of quizzes passed \\
\hline no_quiz_faild & Number of quizzes failed \\
\hline messg_sent_chat & Messages sent to chat \\
\hline messg_sent_teacher & Messages sent to teacher \\
\hline messg_sent_forum & Messages sent to forum \\
\hline messg_read_forum & Messages read on forum \\
\hline time_assignment & Time spent on assignment \\
\hline time_quiz & Time spent on quizzes \\
\hline time_forum & Time spent on forum \\
\hline marks & Total Marks obtained \\
\hline
\end{tabular}

\section{RESULTS AND ANALYSIS}

In this section we present the results of applying $\mathrm{k}$ means algorithm to the data consisting of usage data and marks data obtained by learners in different courses including data mining, operating system, data structure, database system, computer organizati-on and automata theory. Clustering is finding groups of objects such that the objects in one group will be similar to one another and different from the objects in another group[34]. The usage data includes quiz marks on various topics of each course. The k-means algorithm divides a collection of objects into a number of clusters which specified by setting the value of $\mathrm{k}$. In table. 2 we can see three cluster namely not active, avg and active which were obtained as a result of applying k-means algorithm to the moodle data. There are number of data mining tools available but we have use weka primarily due to its open source nature which consists of a collection of machine learning algorithms. We have a total of 100 learners in our database. The first cluster has $9 \%$ of total instances and the second cluster is comprises of $29 \%$ of total instances and finally the $3^{\text {rd }}$ cluster has $62 \%$ of total instances. We have considered 19 attributes as they are sufficient to characterize a cluster. Table. 2 shows that, not active cluster is characterized by low quiz marks(7), low number of assignment done(2), very few number of messages sent to teacher(7), low number of messages sent through forums(10), very low quiz grade obtained(2). On the other hand, the second cluster which is avg is characterized by high number of quizzes done(3), high marks obtained(32), high number of assignments done(9), high number of messages sent through forums(72), high number of resources accessed(82), excellent quiz grade obtained(8), high course total(78). The performance of students of cluster avg is in between not active and active cluster. This cluster is characterized by average marks obtained in quiz(14), average number of assignment done(5), average number of messages sent to teacher(33), average number of messages sent using forum(29), average marks obtained in quiz(4). 
Table.2. Results obtained by Applying K-Means Algorithm

\begin{tabular}{|c|c|c|c|c|}
\hline Attribute & $\begin{array}{c}\text { Full data } \\
(100)\end{array}$ & Not active & Avg & Active \\
\hline Quiz1 & 5.7 & 1.5 & 3.5 & 7.4 \\
\hline Quiz2 & 6.2 & 1.7 & 3.6 & 8.0 \\
\hline Quiz3 & 6.5 & 2.1 & 3.9 & 8.3 \\
\hline Quiz4 & 5.9 & 1.9 & 3.6 & 7.5 \\
\hline Quiz_done & 2.8 & 0.2 & 3.1 & 3.1 \\
\hline Quiz_marks & 24.4 & 7.3 & 14.8 & 31.4 \\
\hline Assign_done & 6.7 & 1.8 & 4.5 & 8.4 \\
\hline Time_assign & 8.0 & 2.7 & 4.9 & 10.2 \\
\hline Messg_teacher & 44.3 & 7.0 & 33.3 & 54.9 \\
\hline $\begin{array}{l}\text { Messg_forum } \\
\text { Forum }\end{array}$ & 53.5 & 10.4 & 29.4 & 71.0 \\
\hline Messg_read & 65.0 & 16.5 & 36.1 & 85.6 \\
\hline Messg_chat & 51.5 & 8.7 & 28.8 & 68.2 \\
\hline Time_quiz & 10.8 & 4.4 & 5.7 & 14.2 \\
\hline Time_forum & 16.6 & 4.6 & 6.2 & 23.3 \\
\hline Access_resours & 70.6 & 51.2 & 46.7 & 84.6 \\
\hline Num_Acess $<30$ & 7.6 & 4.2 & 8.2 & 7.8 \\
\hline Resources_Visit & 38.0 & 21.8 & 39.1 & 49.2 \\
\hline Quiz_grade & 6.1 & 1.8 & 3.7 & 7.9 \\
\hline Course_total & 64.6 & 23.9 & 49.5 & 77.6 \\
\hline
\end{tabular}

\section{CONCLUSION AND FUTURE WORK}

We have proposed an architecture for the recommendation of courses based on their profile. The profile is created using k-means algorithm. We have used weka in order to achieve this objective. Although data of six courses is available, initially we have considered only one course which is data mining and warehousing in order to learn the students' learning behavior/pattern. The application of k-means to moodle's data categorized students in three categories namely active, average and non active. Based on this information we have got some idea of the level of knowledge of students in a particular course such as data mining which helps us to recommend them most appropriate courses. For example, it would be useful for learners of cluster0 to recommend introductory data mining course, as they have shown poor performance in this subject rather than recommending them advance courses of the same subject which might lead to further drop in their performance. Moreover additional knowledge about the learners will help us to improve the recommendation process. Our future work involves evaluating the quality of clusters. Moreover we are also interested in finding those learners, who are less active in collaborative platforms. This means those learners belonging to multiple clusters.

\section{REFERENCES}

[1] C.Romero,S.Ventura, and E.Garcia,"Data mining in course management system: moodle case study and tutorial",Comput.Educ, pp368-384,2008.
[2] V.Kumar, "An Empirical study of the applications of data mining techniques in higher education", International journal of advanced computer science and application, Vol. 2, No.3,pp. 80-84,2011.

[3] Thaiklang,S., Archint,N.,Archint,S., "Learning resource recommendation framework using rule based reasoning approach", Journal of Theoretical and Applied information technology, Vol.69,No.1,2014.

[4] Romero,C., Ventura,S., Delgado, A,J., Bra, P., "Personalized links recommendations based on data mining in adaptive educational hypermedia systems", European Conference on Technology Enhanced Learning,Springer,pp.292-306,2007.

[5] Leony,D.,Pardo,A.,Parada,H., kloos,G. G., "a cloud based architecture for an affective recommender system of e learning resources", $1^{\text {st }}$ international workshop on cloud education environment,2012.

[6] M-S.Chen,., Han,J.,Yu,P.S, "Data mining: An overview from a database perspective", IEEE Transaction on Knowledge and Data Engineering,Vol. 8, pp 866$833,1996$.

[7] Poorni,G.,Balaji,K.,Deeptinivetha,C., “A Personalized ELearning Recommender System using the concept of Fuzzy tree matching",International journal of advanced research in computer engineering and technology,vol.4,no.11,2015.

[8] Daomin,X.,Mingchui,D., "Appropriate learning resource recommendation in intelligent web-based educational system", in proceedings of Intelligent system design and engineering applications,IEEE,2014.

[9] Lopez, B. M., Ramirez, V. R., Hernendez, A, G., “An architecture for developing Educational Recommender Systems", Research in Computing Science,pp.17-26,2015.

[10] Li,X.,Chang,K,S., “A personalized E-learning system based on user profile constructed using information 
fusion", In Proceeding of the 11th International Conference on Distributed Multimedia Systems, pp. 109114, Banff, Canada, Sep. 2005.

[11] Ivanovic, M., Budimac,Z., “Applying recommender system and adaptive hypermedia for E-Learning Personalization", computing and infomatics, vol.32,pp.629-659,2013.

[12] T.Sivakumar., "Learning resources recommendation framework for moodle based on analysis of mostly accessed resources by good students"(Master thesis),retrieved from Digital library university of moratuwa.(http://dl.lib.mrt.ac.lk/handle/123/12347),2014

[13] F.Castro,A.Vellido,A. Nebot, and F.Mugica, "Applying data mining techniques to e-learning problems", Studies in Computational Intelligence, Vol. 62, pp 183-221,2007.

[14] Todtli,B., Laner,M., Semenov,J., Paoli,B., "Recommending Physics Exercise in Moodle Based on Hierarchical Competence Profile", In: Verbert K., Sharples M., Klobučar T. (eds) Adaptive and Adaptable Learning. EC-TEL 2016. Lecture Notes in Computer Science, vol 9891. Springer, Cham.

[15] Ray,S.,Sharma,A., "A collaborative filtering based approach for recommending elective courses", In Proceedings of the 5th International Conference on Information Intelligence, Systems, Technology and Management (ICISTM), S. Dua, S. Sahni, and D. Goyal, Eds. Springer-Verlag, 330-339,2011.

[16] Tang,Y,T., McCalla,G., "smart recommendation for an evolving e-learning system", Workshop on Technologies for Electronic Documents for Supporting Learning, International Conference on Artificial Intelligence in Education,pp.699-710,2003.

[17] Pallavi,S., Chavan,A., "Open-source learning management system with moodle",2004.

[18] Al-Radaideh,E.AI-Shawakfa, \& AI-Najjar, “ Mining students data using decision tree", In proceedings of the international Arab conference on information technology,2006.

[19] Lu,J., "A personalized e-learning material recommender system", International Conference on Information Technology, Macquarie Scientific Publishing,2004.

[20] Carmona,C.,Castillo,G.,Millan,E., "Discovering Student Preferences in E-learning", Proceedings of the International Workshop on Applying Data Mining in eLearning,pp.33-42,2007.

[21] Salehi,M.,Kamalabadi,N,I.,Ghoushchi,G, B,M., "Personalized recommendation of learning material using sequential pattern mining and attribute based collaborative filtering", Education and information technology,vol.19,no.4,pp.713-735,2014.

[22] Sin,K.,Muthu,L., "Application of big data in education data mining and learning analytics-A literature review",ITACT journal on soft computing,vol.5,no.4,2015.

[23] Baker, R.S.J.d. (in press) Data Mining for Education. To appear in McGaw, B., Peterson, P.,Baker, E. (Eds.) International Encyclopedia of Education (3rd edition). Oxford, UK: Elsevier.

[24] Algarni,A., "Data mining in Education", International journal of Advanced Computer Science and Applications,Vol.7,No.6,2016.

[25] Romero,C.,Ventura,S., "Data mining in education", WIREs Data Mining Knowl Discovery, Vol.3,pp. 1227,2013.

[26] Namratha,B., "Educational Data Mining-Applications and Techniques", International journal of latest trends in Engineering and Technology,Vol.7,No.2,2016.
[27] Koedinger, K. R., D'Mello, S., McLaughlin, E. A., Pardos, Z. A.,Rosé, C. P.,. "Data mining and education", Wiley Interdisciplinary Reviews: Cognitive Science. Vol.6,No. 4, pp. 333-353,2015.

[28] Feng,M.,Bienkowski,M., Means,B.,"Enhancing Teaching and Learning through EducationalData Mining and Learning Analytics: An Issue Brief",U.S. Department of Education, 2012.

[29] Merceron,A.,Yacef,K., "Educational data mining: A case study”, In proceedings of AIED,pp.467-474,,2005.

[30] Tzouveli, P., Mylonas, P.,Kollias, S., “An Intelligent ELearning System Based on Learner Profiling and Learning Resources Adaptation", Computers \& Education, Vol.51,No.1, pp.224-238,2008.

[31] Romero, C., Ventura, S., Delgado, J. A., Bra, P. D. (in press). Personalized links recommendation based on data mining in adaptive educational hypermedia systems, In Second European Conference on technology enhanced learning. Crete, Greece.

[32] E.Frank, A.Mark A. Hall, H.Ian, and H-I Witten, "Data Mining: Practical Machine Learning Tools and Techniques", Morgan Kaufmann, Fourth Edition,2016.

[33] C.Romero,S.Ventura,P. G, Espejo,C. Hervas, "Data mining algorithms to classify students",In: Education Data Mining conference, 2008.

[34] Aher,S.B.,Lobo,L., "Combination of machine learning algorithms for recommendation of courses in E-learning system based" on historical data.", Knowledge-Based System, Elsevier,Vol.51, pp.1-14,2013.

[35] Mueen,A.,Zafar,B.,Manzoor,U., "Modeling and "Predicting Students' Academic Performance Using Data Mining Techniques", International Journal of Modern Education and Computer Science, MECS, Vol.8,No.11,pp.36-42,2016.

[36] Da,M.,Wei,W.,Hai-gauan,H.,Jian-he,G., "The Application of Bayesian Classification Theories in Distance Education System", Internationl Journal of Modern Education and Computer Science,MECS,Vol.4,pp.9-16,2011.

\section{Authors' Profiles}

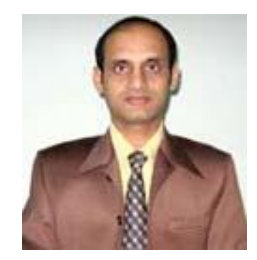

Prof. Sanjay K. Dwivedi is working as Professor \& Head, Department of Computer science at Babasaheb Bhimrao Ambedkar Central university,Lucknow,India. His research interest includes Artificial intelligence,Information retrieval, Web mining,NLP and WSD. He has published number of research papers in reputed journals and conferences. He is approachable atskd200@yahoo.com.

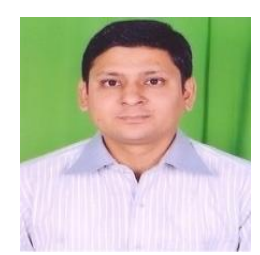

Bhupesh Rawat received his M.C.A degree from H.N.B Garhwal University Srinagar, Uttrakhand, India and is pursuing his Ph.D in the Department of Computer Science at Babasaheb Bhimrao Ambedkar Central University, Lucknow, India. His major research interest includes data mining, fuzzy logic and semantic web. He has published papers in various international journals and conferences. $\mathrm{He}$ is approachable at bhr222@gmail.com. 
How to cite this paper: Bhupesh Rawat, Sanjay K. Dwivedi, "An Architecture for Recommendation of Courses in E-learning System", International Journal of Information Technology and Computer Science(IJITCS), Vol.9, No.4, pp.39-47, 2017. DOI: 10.5815/ijitcs.2017.04.06 